\title{
FUNGOS MICORRÍZICOS ARBUSCULARES EM SERINGUEIRA EM LATOSSOLO AMARELO DISTRÓFICO DA AMAZÔNIA OCIDENTAL ${ }^{1}$
}

\author{
Larissa Alexandra Cardoso Moraes², Luadir Gasparotto²e Adônis Moreira²
}

\begin{abstract}
RESUMO - A colonização radicular com fungos micorrízicos arbusculares (FMA) pode aumentar a eficiência no crescimento e absorção de nutrientes pelas plantas. Com o objetivo de verificar esse efeito, foram avaliados o grau de colonização em seringais adultos cultivados em Latossolo Amarelo distrófico e a eficiência de seis FMAs na colonização, crescimento e estado nutricional de mudas de seringueira com três e seis meses de transplantio. Os resultados indicaram baixo grau de colonização micorrízica e número de esporos em seringal adulto. Seis meses depois do transplantio das mudas de seringueira foram suficientes para ocorrer colonização de FMAs detectável. Não houve aumento no incremento em altura, diâmetro e emissão de folhas, independentemente do inóculo utilizado. Os teores foliares de nutrientes (N, P, K, Ca, Mg, Cu, Fe, Mn e Zn) também não foram influenciados pelo número de esporos e grau de colonização, havendo diferenças apenas em função da idade das plantas.
\end{abstract}

Palavras-chave: Hevea sp, Estado nutricional e Nutrientes.

\section{ARBUSCULAR MYCORRHIZAE FUNGI, GROWTH AND NUTRIENT CONTENT IN RUBBER PLANTS IN A XANTHIC FERRASOL OF WESTERN AMAZON}

\begin{abstract}
The infection roots with arbuscular mycorrhizal fungi (AMF) can increase the efficiency in growth and nutrients uptake of plants. With the objective to verify this effect, the degree of colonization in rubber tree plantation cultivated in a Xanthic Ferralsol (dystrophic Yellow Latosol) and the efficiency of six AMF in colonization, growth and nutritional status of rubber tree seedlings were evaluated, with three and six months of transplanting. The results showed a low level of mycorrhizal infection and number of spores in adult rubber tree. Six months of transplanting of rubber tree seedlings were sufficient detectable AMFs infection. There was no increase in height, diameter and number of leaves. The foliar nutrients concentration $(N, P, K, C a, M g, C u, F e, M n$ and $\mathrm{Zn}$ ) were also not influenced by the number of spores and infection degree, with only differences by age of the plants (three and six months of transplanting).
\end{abstract}

Keywords: Hevea sp., nutritional status and nutrients.

\section{INTRODUÇÃO}

Causas edafoclimáticas fazem com que a Amazônia tropical úmida geralmente apresente limitações para o desenvolvimento da agricultura. Os principais fatores para que isso ocorra são: baixa fertilidade do solo, com elevada acidez e baixa soma de bases (SANCHEZ et al., 1982; MOREIRA e FAGERIA, 2009), alta umidade e elevada temperatura da região que favorecem a infestação de doenças fúngicas. No caso da seringueira (Hevea brasiliensis), a presença do fungo Microcyclus uley (mal-das-folhas) reduz significativamente a produção de látex (GASPAROTTO et al., 1997).

Apesar desses problemas, em termos de fertilidade, uma característica importante do cultivo da seringueira é que o látex apresenta na sua composição de 91 a 96\% de hidrocarboneto (MORTON, 1989); consequentemente, a exportação de N, P, K, Ca e Mg é muito baixa quando comparada com outros cultivos tropicais (MOREIRA et al., 2009). No entanto, a ocorrência

\footnotetext{
${ }^{1}$ Recebido em 09.05.2008 e aceito para publicação em 02.03.2010.

${ }^{2}$ Embrapa Amazônia Ocidental, Manaus, AM, Brasil. E-mail: <larissa.moraes@cpaa.embrapa.br>, <gasparotto@cppa.embrapa.br> e <adonis.moreira@cpaa.embrapa.br>.
} 
do mal-das-folhas, que dificultou sua expansão, atualmente pode ser controlada com o uso da enxertia de copa com espécies de Hevea resistentes à doença (MORAES e MORAES, 2008).

Apesar de sua rusticidade, na fase imatura da seringueira o uso de fertilizantes e corretivos da acidez se faz necessário, para que ocorra crescimento vegetativo mais rápido até o fechamento do dossel e início da sangria. Em Latossolo Amarelo distrófico da Amazônia, no preparo da área, deve-se aplicar uma tonelada de calcário por hectare (MORAES, 1984), e na fase de viveiro Pereira et al. (1986) recomendaram a aplicação de $150 \mathrm{~kg} \mathrm{ha}^{-1}$ de $\mathrm{P}_{2} \mathrm{O}_{5}$ e $60 \mathrm{~kg} \mathrm{ha}^{-1}$ de $\mathrm{K}_{2} \mathrm{O}$. Nesse caso, o uso de pequenas quantidades de insumos pode aumentar a eficiência de absorção de nutrientes pelas plantas com a utilização de fungos micorrízicos arbusculares (FMAs), em razão do menor diâmetro das hifas, aumentando o volume de solo explorado (WALLANDER et al., 2002).

Na Amazônia, grande número de espécies apresenta colonização natural por FMAs (OLIVEIRA e OLIVEIRA, 2005), existindo relatos de ocorrência associados ao gênero Hevea brasiliensis em condições naturais, cuja colonização é inicialmente formada por outros fungos, como: Rhizophagus e tipos de Rhizoctonia e, posteriormente, somente por Rhizophagus (WASTIE, 1965).

A seringueira é um das espécies arbóreas da Amazônia naturalmente infectadas pelos FMAs (TAWARAYA et al., 2003). Porém, existem poucas informações sobre os efeitos que essa associação possa trazer para a cultura, havendo, nesse caso, resultados positivos em crescimento e diâmetro do caule, como os obtidos por Diniz et al. (2010) com Glomus clarum, e também a ausência de resposta, como os encontrados por Lopes e Cardoso (2001).

Buscando verificar a eficiência de diferentes FMAs no crescimento e absorção de nutrientes, o objetivo deste trabalho foi avaliar a presença através do número de esporos e da porcentagem de colonização em seringais adultos e o efeito de seis espécies de inóculos de FMAs no crescimento, absorção de nutrientes, estado nutricional, número de esporos e porcentagem de colonização em mudas de seringueira com três e seis meses de transplantio.

\section{MATERIAL E MÉTODOS}

Para realização desses estudos, foram feitos dois experimentos: a) condições de campo em Latossolo Amarelo distrófico; e b) casa de vegetação, ambos realizados na área experimental da Embrapa Amazônia Ocidental, localizada no Município de Manaus, nas coordenadas geográficas 38' S e 5952’ W, Estado do Amazonas. O clima dominante no local é tropical úmido tipo Afi pela classificação de Köppen, apresentando chuvas relativamente abundantes durante todo o ano (média de $2250 \mathrm{~mm}$ ), e a quantidade de chuva no mês de menor precipitação é sempre superior a $60 \mathrm{~mm}$. A temperatura média anual da região é de aproximadamente $26^{\circ} \mathrm{C}$ (VIEIRA e SANTOS, 1987).

\section{a) Experimento de campo}

Foram utilizados um seringal nativo e oito seringais com diferentes tipos de manejo para coleta das amostras de solo, sendo: três seringais com enxertia de copa (copa de Hevea brasiliensis ou de Hevea pauciflora - denominados no estudo como 1,2 e 3 ) e cinco de $H$. brasiliensis - denominados como 4, 5, 6, 7 e 8 .

Nesses seringais, foram coletadas na camada de 0-10 cm de profundidade, dez amostras de solo por área e 10 de raízes adventícias por planta em cada área. Para quantificar a densidade de esporos de fungos micorrízicos vesicular-arbusculares (FMA), foram utilizados $50 \mathrm{~g}$ de solo. Nas raízes, foram determinadas a porcentagem de colonização micorrízica e a presença de arbúsculos e hifas totais extracelulares (FMAS e de outros fungos de solo) com escalas, sendo classificadas e divididas em quatro faixas de presença: alta (70-100\%), média (40-69\%), baixa (1-39\%) e ausente (0\%).

As amostras de solo também foram submetidas às análises químicas e físicas (EMBRAPA, 1997). Após a secagem ao ar e passadas em peneira de malha de 4,0 mm, as análises de solo consistiram nas seguintes determinações: $\mathrm{pH}\left(\mathrm{H}_{2} \mathrm{O}\right)$, $\mathrm{P}$ e $\mathrm{K}$ disponível (Mehlich 1), Ca, Mg e Al trocável ( $\mathrm{KCl} 1,0 \mathrm{~mol} \mathrm{~L}^{-1}$ ), granulometria (areia, silte e argila) e densidade do solo (Tabela 1).

\section{Extração e quantificação de esporos}

Os esporos foram extraídos por decantação e peneiramentoúmido(GERDEMANN eNICOLSON, 1963), seguidos por centrifugação em água e em sacarose 40\% (JENKINS, 1964). A extração e quantificação de esporos se deram a partir de $50 \mathrm{~g}$ de terra destorroadaem 
Tabela 1 - Análise química e física do solo dos seringais nativo e cultivados.

Table 1 - Soil chemical and physical analysis in native and cultivated rubber plants.

\begin{tabular}{|c|c|c|c|c|c|c|c|c|c|c|}
\hline \multirow[t]{2}{*}{ Áreas } & \multirow{2}{*}{$\begin{array}{c}\mathrm{pH} \\
\text { água }\end{array}$} & $\mathrm{P}$ & $\mathrm{K}$ & $\mathrm{Ca}$ & $\mathrm{Mg}$ & $\mathrm{Al}$ & Areia & Silte & Argila & \multirow{2}{*}{$\begin{array}{c}\text { Ds } \\
\mathrm{g} \mathrm{cm}^{-3}\end{array}$} \\
\hline & & \multicolumn{2}{|c|}{$\overline{-\mathrm{mg} \mathrm{dm}^{-3}-二}$} & \multicolumn{3}{|c|}{$-\mathrm{cmol}_{\mathrm{c}} \mathrm{dm}^{-3}-\square$} & \multicolumn{3}{|c|}{$\longrightarrow \mathrm{g} \mathrm{kg}^{-1}-{ }_{-}$} & \\
\hline Seringal Nativo & 3,6 & 5 & 39 & 0,07 & 0,20 & 3,33 & 200 & 200 & 600 & 1,05 \\
\hline Seringal $1^{(1)}$ & 3,9 & 37 & 37 & 0,31 & 0,14 & 1,73 & 180 & 90 & 630 & 1,30 \\
\hline Seringal 2 & 4,4 & 126 & 81 & 1,22 & 0,63 & 1,40 & 290 & 10 & 700 & 0,97 \\
\hline Seringal 3 & 4,2 & 112 & 87 & 0,79 & 0,81 & 1,67 & 200 & 100 & 700 & 1,20 \\
\hline Seringal 4 & 5,0 & 155 & 90 & 1,78 & 0,82 & 1,01 & 150 & 200 & 650 & 1,10 \\
\hline Seringal 5 & 4,8 & 15 & 73 & 2,04 & 0,75 & 1,10 & 210 & 90 & 700 & 1,30 \\
\hline Seringal 6 & 3,8 & 17 & 57 & 1,47 & 0,62 & 2,73 & 140 & 110 & 750 & 1,13 \\
\hline Seringal 7 & 3,9 & 55 & 55 & 0,48 & 0,19 & 2,17 & 180 & 180 & 640 & 1,35 \\
\hline Seringal 8 & 3,5 & 59 & 59 & 0,27 & 0,16 & 2,10 & 230 & 160 & 610 & 1,43 \\
\hline Média & 4,1 & 65 & 60 & 0,94 & 0,48 & 1,92 & 198 & 138 & 664 & 1,20 \\
\hline
\end{tabular}

(1) Seringais 1, 2 e 3 com enxertia de copa (copas de Hevea brasiliensis ou Hevea pauciflora) e 4, 5, 6, 7 e 8 seringais de H. brasiliensis.

(1) Rubber plants 1, 2 and 3 with crown grafting (crown of Hevea brasiliensis or Hevea pauciflora) and 4, 5, 6, 7 and 8 rubber plants of $H$. brasiliensis.

2L litros de água e decantadas após passar em peneira de malha de $0,84 \mathrm{~mm}$, sendo o resíduo retido descartado. A suspensão foi passada em peneira de $0,044 \mathrm{~mm}$, para retenção dos esporos. Os esporos retidos foram centrifugados durante $5 \mathrm{~min}$ a $1.750 \mathrm{rpm}$. O sobrenadante foi eliminado, adicionando-se sobre os esporos a solução de sacarose $40 \%$, e novamente centrifugado por $1 \mathrm{~min}$ na mesma rotação anterior. Em seguida, o sobrenadante foi vertido numa peneira de 0,044 $\mathrm{mm}$ e enxaguado abundantemente. Os esporos foram retirados e a contagem, realizada em câmera de contagem de Peter.

\section{Método de coloração das raízes e avaliação da incidência de colonização micorrízica}

As amostras de raízes adventícias coletadas na camada de 0-10 cm de profundidade nas nove áreas foram lavadas e colocadas em solução de KOH 10\% para clareamento, permanecendo nessa solução por 5 min. Em seguida, foram lavadas e mantidas durante 48 h, em lactofenol com azul de algodão 0,05\%. Posteriormente, as raízes foram lavadas para determinação da incidência de colonização micorrízica (PHILLIPS e HAYMAN, 1970; SILVA et al., 2007a).

A porcentagem de incidência de colonização micorrízica foi determinada sob microscópio estereoscópico, com o auxílio de uma placa de Petri com arranjo quadriculado (GIOVABBETI e MOSSE, 1980). Cada amostra foi espalhada sobre a placa para realização da contagem do número de radicelas com e sem colonização.

\section{b) Experimento em casa de vegetação}

O experimento de casa de vegetação foi realizado utilizando-se o solo de 40-60 cm sob floresta primária (Latossolo Amarelo distrófico). O solo depois de coletado foi passado em peneira de malha de 4,0 mm e esterilizado duas vezes em autoclave a $120^{\circ} \mathrm{C}$, durante 3 h. Após a autoclavagem, foi feita a calagem com calcário dolomítico, usando-se um quantidade correspondente a uma tonelada por hectare e incubada por 15 dias em sacos plásticos com 9 kg de capacidade (MORAES, 1984).

Na inoculação foram utilizadas seis espécies de fungos micorrízicos provenientes do Laboratório de Microbiologia do Instituto Nacional de Pesquisas da Amazônia (INPA-Manaus), sendo estes: Glomus macrocarpum, Glomus claroideum, Glomus etunicatum, Gigaspora margarita, Scutellospora gregaria e Scutellospora margarita. O delineamento experimental utilizado foi o inteiramente casualizado com sete tratamentos (seis inóculos mais a testemunha) e cinco repetições.

Sementes ilegítimas de Hevea brasiliensis foram semeadas em substrato composto por areia e serragem esterilizadas, proporção v:v (1:1). As mudas permaneceram nesse substrato até atingirem cerca de $10 \mathrm{~cm}$ de altura e apresentarem um par de folhas, sendo posteriormente transplantadas para sacos plásticos contendo o solo autoclavado e adubado. Foram utilizadas duas mudas uniformes de seringueira por saco. Antes 
do plantio, foram misturados na camada de 0-7 cm cerca de 600 esporos e raízes de arroz infectadas com fungos micorrízicos. Os sacos foram irrigados diariamente com água destilada por um período de seis meses (agosto a janeiro).

Após três meses do plantio foi colhida uma planta de cada repetição, bem como determinadas as seguintes variáveis: diâmetro do caule $(\mathrm{mm})$, número de lançamentos, número de folhas, altura das plantas $(\mathrm{cm})$, colonização de FMAs nas raízes (\%) e número de esporos. No sexto mês foi retirada outra planta e analisadas as mesmas variáveis.

Nas duas épocas foram coletadas as folhas recémmaduras para análise foliar. Após a coleta, as folhas foram secas em ventilação forçada em estufa a $\pm 65^{\circ}$ $C$ até peso constante e passadas em moinho com peneira de $0,40 \mathrm{~mm}$. O N total foi extraído por digestão sulfúrica e determinado pelo método Micro-Kjeldahl (NELSON e SOMMERS, 1972), Os teores de P, K, Ca, Mg, Cu, Fe, Mn e Zn total foram extraídos por digestão em solução nitricoperclórica. O P total foi determinado por espectrofotometria com azul-de-molibdênio (NOVOZAMSKY et al., 1983). O K, Ca, Mg, Cu, Fe, Mn e Zn total foram analisados por espectrofotômetria de absorção atômica, de acordo com os métodos descritos por Walinga et al. (1995).

De acordo com o delineamento proposto, os resultados foram submetidos à análise de variância (ANOVA), teste F e comparação de médias com o teste de Tukey a 5\% de significância (PIMENTEL GOMES e GARCIA, 2002).

\section{RESULTADOS E DISCUSSÃO}

Os resultados de análise de solo dos seringais nativos e cultivados mostraram acidez elevada, com altos teores de Al trocável (Tabela 1 ). Os teores de $\mathrm{P}$ disponível ficaram acima de $12,0 \mathrm{mg} \mathrm{kg}^{-1}$, valores considerados elevados para solos com 600 a $1.000 \mathrm{~g}$ $\mathrm{kg}^{-1}$ de argila (ALVAREZ VENEGAS et al., 1997). Com relação aos teores de K disponível e Ca e Mg trocáveis, nos seringais, esses valores ficaram, na média, em 60 $\mathrm{mg} \mathrm{kg}^{-1}, 0,94 \mathrm{cmol}_{\mathrm{c}} \mathrm{dm}^{-3} \mathrm{e}$ 0,48 $\mathrm{cmol}_{\mathrm{c}} \mathrm{dm}^{-3}$. Esses valores são considerados na classe de interpretação de fertilidade do solo como médio, baixo e médio, respectivamente (ALVAREZ VENEGAS et al., 1997). Exceto o seringal nativo, a fertilidade do solo observada nos seringais cultivados (Tabela 1) difere, em parte, dos encontrados por Moreira e Fageria (2009), nos quais verificaram que os solos cultivados ou não do Estado do Amazonas são caracterizados, na sua maior parte, pela acidez elevada e baixa fertilidade do solo, com $\mathrm{H}^{+}+\mathrm{Al}^{3+}$ predominando no complexo de troca. O aumento da fertilidade nos seringais cultivados possivelmente se deva ao manejo adotado para sua implantação

O número de esporos e a porcentagem de colonização das raízes nos seringais cultivados e nativos são mostrados na Tabela 2. Verificou-se que o número de esporos variou de 108 a 1.213, enquanto a colonização micorrízica foi de 6,15 a 41,23\%, sendo os menores valores observados no seringal nativo, apresentando diferenças significativas (pd”0,05) com os seringais cultivados. Exceto no seringal nativo (6,15\%), os valores dos seringais cultivados estão próximos dos encontrados por Oliveira et al. (1999) em oito espécies florestais nativas sob Argissolo distrófico nas mesmas condições climáticas. Outro motivo para a baixa colonização e número de esporos foi a qualidade (poucos pelos radiculares ou raízes secundárias) e quantidade (baixas) de raízes presentes no volume de solo típica da seringueira.

Nos seringais cultivados, a média do número de esporos em $50 \mathrm{~g}$ de solo e grau de colonização foi de 813,0 e $29,5 \%$. Fatores como a característica do sistema radicular da planta, com baixa ocorrência de FMAs, também foram verificadas em áreas cultivadas com seringueira na Malásia (IKRAM e MAHMUAD, 1984), onde a porcentagem de colonização micorrízica ficou na faixa de 0 a $50 \%$, Aliado a isso, os altos teores de $\mathrm{P}$ disponível no solo $(0-20 \mathrm{~cm})$ dos oito seringais cultivados (Tabela 1) podem ter interferido negativamente na colonização radicular (SIQUEIRA, 1996). Apesar do baixo grau de colonização, esses valores foram 7,5 e 4,8 vezes maiores que o encontrado no seringal nativo.

No caso da presença de arbúsculos e das hifas extracelulares (Tabela 1), exceto nos seringais 1 e 2 , foi observada nas demais áreas ausência nas raízes de hifas extracelulares, enquanto nos arbúsculos as quantidades ficaram dentro das faixas indicadas como de média e baixa presenças. Essa ausência de hifas, responsável pelo aumento da área de superfície de contato das raízes com o solo (MARSCHNER e DELL, 1994), associada à pequena presença de arbúsculos, devem estar relacionadas às características das plantas de seringueira, que exportam basicamente hidrocarbonetos 
Tabela 2 - Número de esporos de FMA em 50 gramas de solo, porcentagem de colonização micorrízica e presença de arbúsculos e hifas extracelulares nos seringais nativo e cultivados ${ }^{(1)}$

Table 2 - Number of spores of AMF in 50 grams of soil, percentage of mycorrhizal colonization and the presence of arbuscules and extracellular hyphae in native and cultivated rubber plants.

\begin{tabular}{|c|c|c|c|c|}
\hline \multirow[t]{2}{*}{ Áreas } & \multirow{2}{*}{$\begin{array}{l}\text { Esporos de FMA } \\
\text { em } 50 \mathrm{~g} \text { de solo } \\
\mathrm{n}\end{array}$} & \multirow{2}{*}{$\begin{array}{l}\text { Colonização micorrízica } \\
\begin{array}{l}\text { \% } \%\end{array}\end{array}$} & Arbúsculos & Hifas extracelulares \\
\hline & & & —— & nça —— \\
\hline Seringal Nativo & $108 \mathrm{c}$ & $6,15 f$ & ++ & + \\
\hline Seringal $1^{(2)}$ & $643 b$ & $31,40 \mathrm{bc}$ & ++++ & +++ \\
\hline Seringal 2 & 1388a & $41,23 a$ & +++ & ++++ \\
\hline Seringal 3 & 520b & $25,32 \mathrm{~cd}$ & ++ & + \\
\hline Seringal 4 & 464b & $18,30 \mathrm{e}$ & ++ & + \\
\hline Seringal 5 & 466b & 20,00de & +++ & + \\
\hline Seringal 6 & $1184 a$ & $35,25 a b$ & +++ & + \\
\hline Seringal 7 & $1213 a$ & $36,07 \mathrm{ab}$ & ++ & + \\
\hline Seringal 8 & $628 b$ & $28,31 c$ & +++ & + \\
\hline Média & 735 & 26,56 & & \\
\hline
\end{tabular}

(1) Médias seguidas por letras distintas na mesma coluna diferem entre si a 5\% de probabilidade pelo teste de Tukey.

(2) Seringais 1, 2 e 3 com enxertia de copa (copa de Hevea brasiliensis ou Hevea pauciflora) e 4, 5, 6, 7 e 8 seringais de H. brasiliensis.

++++ (alta), +++ (média), ++ (baixa) e + (ausência) de arbúsculos e hifas extracelulares.

(1) Means followed by different letters in the same column differ at 5\% probability by Tukey's test.

(2) Rubber plants 1, 2 and 3 with crown grafting (crown of Hevea brasiliensis or Hevea pauciflora), 4, 5, 6, 7 and 8 rubber tree $H$. brasiliensis.

++++ (High),+++ (medium),++ (low) and + (absence) of extracellular hyphae and arbuscules.

(MOREIRA et al., 2009), Consequentemente, a absorção e exportação de N, P, K, Ca e Mg em seringais adultos são muito baixas. Outro problema para os baixos valores encontrados na extração de hifas é a textura pesada dos solos (664 $\mathrm{g} \mathrm{kg}^{-1}$ de argila), o que dificultou a quantificação destes.

No experimento realizado em condições de casa de vegetação, verificou-se nas duas avaliações que o diâmetro do caule, número de lançamentos, número de folhas e altura das plantas não foram estatisticamente influenciados pelos tipos de inóculo, havendo diferenças apenas em função da idade (Tabela 3). A ausência de resposta para essas variáveis também foi reportada por Wastie (1965) após 18 meses de inoculação, por Lopes e Cardoso (2001) em mudas com nove meses cultivadas em casa de vegetação com Neossolo Quartzarênico e Pereira et al. (1986) em viveiro em condições de campo sobre um Latossolo Amarelo distrófico. Nesses dois últimos casos, o teor de $\mathrm{P}$ disponível no solo era muito baixo (4,0 $\mathrm{mg} \mathrm{kg}^{-1}$ - extrator resina e 3,0 $\mathrm{mg} \mathrm{kg}^{-1}$ - extrator Mehlich 1, respectivamente), o que demonstra a eficiência da seringueira na absorção de nutrientes em áreas de baixa fertilidade do solo.
Quanto ao número de esporos e à porcentagem de colonização micorrízica nas duas épocas de avaliação (Tabela 4), apesar da ausência de colonização, verificou-se que, exceto o Glomus etunicatum na primeira avaliação e o Glomus claroideum na segunda, houve incremento significativo do número de esporos por planta em relação à testemunha. Porém, dentro da própria espécie houve grande variação na taxa de incremento com redução de $67 \%$ no Glomus claroideum e aumento de $400 \%$ no Glomus etunicatum do terceiro para o sexto mês de replantio. Independentemente da ausência de colonização micorrízica e devido, possivelmente, ao baixo número de esporos, o aumento de Glomus etunicatum se torna relevante, haja vista que estudos com clones de seringueira inoculados com essa espécie (FELDMANN et al., 1988) apresentaram maior crescimento e resistência ao Microcyclus ulei, principal patógeno da cultura (GASPAROTTO et al., 1997). Cabe destacar que a baixa colonização pode ser devida, também, ao elevado teor de Al trocável no solo do substrato (Tabela 1), inibindo a atuação dos FMAs no crescimento das plantas (Tabela 3).

Revista Árvore, Viçosa-MG, v.34, n.3, p.389-397, 2010 
Tabela 3 - Influência dos FMAs no diâmetro do caule, número de lançamentos, número de folhas e altura de mudas de seringueira aos três e seis meses após o transplantio ${ }^{(1)}$.

Table 3 - Influence of AMF in stem diameter, number of releases, number of leaves and plant height of rubber seedling at three and six months after transplant ${ }^{(1)}$.

\begin{tabular}{|c|c|c|c|c|c|c|c|c|}
\hline \multirow[t]{3}{*}{ Tratamentos } & \multicolumn{2}{|c|}{ Diâmetro do caule } & \multicolumn{2}{|c|}{ Lançamentos } & \multicolumn{2}{|c|}{ Folhas } & \multicolumn{2}{|c|}{ Altura } \\
\hline & três & seis & três & seis & três & seis & três & seis \\
\hline & \multicolumn{2}{|c|}{$-\mathrm{cm}=-$} & & & & & \multicolumn{2}{|c|}{$-(\mathrm{cm}=-$} \\
\hline G. macrocarpum & $0,55 b$ & $0,88 a$ & $2 b$ & $5 a$ & $8 \mathrm{~b}$ & $19 \mathrm{a}$ & $49 b$ & $142 \mathrm{a}$ \\
\hline G. claroideum & $0,56 b$ & $0,87 a$ & $2 b$ & $5 a$ & $8 b$ & $19 a$ & $50 \mathrm{~b}$ & $144 a$ \\
\hline G. etunicatum & $0,55 b$ & $0,93 a$ & $1 \mathrm{~b}$ & $5 a$ & $8 b$ & $18 \mathrm{a}$ & $51 b$ & $154 a$ \\
\hline G. margarita & $0,56 b$ & $0,92 \mathrm{a}$ & $2 b$ & $5 a$ & $8 b$ & $18 \mathrm{a}$ & $55 b$ & $147 \mathrm{a}$ \\
\hline S. gregaria & $0,54 b$ & $0,87 a$ & $2 b$ & $5 a$ & $8 \mathrm{~b}$ & $19 a$ & $51 \mathrm{~b}$ & $144 a$ \\
\hline S. margarita & $0,54 b$ & $0,87 \mathrm{a}$ & $2 b$ & $5 a$ & $8 b$ & $18 \mathrm{a}$ & $52 b$ & $141 \mathrm{a}$ \\
\hline Testemunha & $0,55 b$ & $0,87 a$ & $2 b$ & $5 a$ & $8 b$ & $18 \mathrm{a}$ & $54 \mathrm{~b}$ & $146 a$ \\
\hline Médias & $0,55 b$ & $0,89 a$ & $2 b$ & $5 a$ & $8 b$ & $18 \mathrm{a}$ & $52 b$ & $145 a$ \\
\hline
\end{tabular}

(1) Médias seguidas por letras distintas na mesma linha dentro de cada tratamento e das médias em cada variável analisada, diferem entre si a 5\% de probabilidade pelo teste de Tukey.

(1) Means followed by different letters in the same row within each treatment and the means of each variable studied, differ at $5 \%$ probability by Tukey's test.

Tabela 4 - Número de esporos por planta e porcentagem de colonização micorrízica aos três e seis meses da inoculação e plantio das mudas de seringueira ${ }^{(1)}$.

Table 4 - Number of spores per plant and percentage of mycorrhizal colonization at three and six months after inoculation and rubber seedlings plantation ${ }^{(1)}$.

\begin{tabular}{|c|c|c|c|c|c|}
\hline \multirow[t]{3}{*}{ Tratamentos } & \multicolumn{2}{|c|}{ Três meses } & \multicolumn{2}{|c|}{ Seis meses } & \multirow[t]{2}{*}{$\ddot{\mathrm{A}}$} \\
\hline & Esporos & Colonização & Esporos & Colonização & \\
\hline & $-\mathrm{n}-$ & $-\%-$ & $\overline{-n--}$ & $-\%=$ & $-\%=$ \\
\hline Glomus macrocarpum & $30 \mathrm{~b}$ & 0 & 40bc & 0 & 25 \\
\hline Glomus claroideum & $60 a$ & 0 & $20 d$ & 0 & -67 \\
\hline Glomus etunicatum & $10 \mathrm{c}$ & 0 & $50 \mathrm{ab}$ & 0 & 400 \\
\hline Gigaspora margarita & $40 \mathrm{~b}$ & 0 & $30 c d$ & 0 & -20 \\
\hline Scutellospora gregaria & $40 \mathrm{~b}$ & 0 & $60 a$ & 0 & 50 \\
\hline Scutellospora margarita & $70 a$ & 0 & 30cd & 0 & -57 \\
\hline Testemunha & $10 c$ & 0 & $20 d$ & 0 & 100 \\
\hline Médias & $37 \mathrm{~A}$ & 0 & $36 \mathrm{~A}$ & 0 & \\
\hline
\end{tabular}

(1) Médias seguidas por letras minúsculas distintas na mesma coluna e maiúsculas dentro das médias, diferem entre si a 5\% de probabilidade pelo teste de Tukey. Ä - variação no número de esporos nas duas avaliações ( $3^{\circ}$ e $6^{\circ}$ mês).

(1) Means followed by small letters in the same column and in case of averages differ at $5 \%$ probability by Tukey's test. $\ddot{A}$ - change in number of spores in two evaluations ( $3^{\text {th }}$ and $6^{\text {th }}$ months).

Os teores foliares de N, P, K, Ca e Mg das plantas inoculadas e não inoculadas não diferiram estatisticamente ( $\mathrm{p}>0,05)$, havendo diferenças do $\mathrm{N}$ e Ca apenas em função das épocas de amostragem (Tabelas 5). Nas mudas de seringueira, o teor de $\mathrm{N}$ variou de 27,6 a 39,1 $\mathrm{g} \mathrm{kg}^{-1}$ e 31,3 a $32,6 \mathrm{~g} \mathrm{~kg}^{-1}$ aos três e seis meses de transplantio, respectivamente. Apesar da ausência de adubação nitrogenada, esses teores ficaram acima dos 26 a $27 \mathrm{~g} \mathrm{~kg}^{-1}$ estabelecidos por Bolle-Jones (1954) para mudas de seringueira. No caso do $\mathrm{P}$, na média das amostragens os teores variaram de 1,4 a 1,6 $\mathrm{g} \mathrm{kg}^{-1}$, não havendo diferenças entre as épocas de amostragens. Lopes e Cardoso (2001) encontraram teores variando de 0,9 a $1,8 \mathrm{~g} \mathrm{~kg}^{-1} \mathrm{em}$ mudas de seringueira inoculadas e não inoculadas com FMAs, sem apresentarem sintomas de deficiência. Para BolleJones (1954), nesse estágio da planta, o teor adequado deve ficar na faixa de 1,6 a 2,3 $\mathrm{g} \mathrm{kg}^{-1}$. 


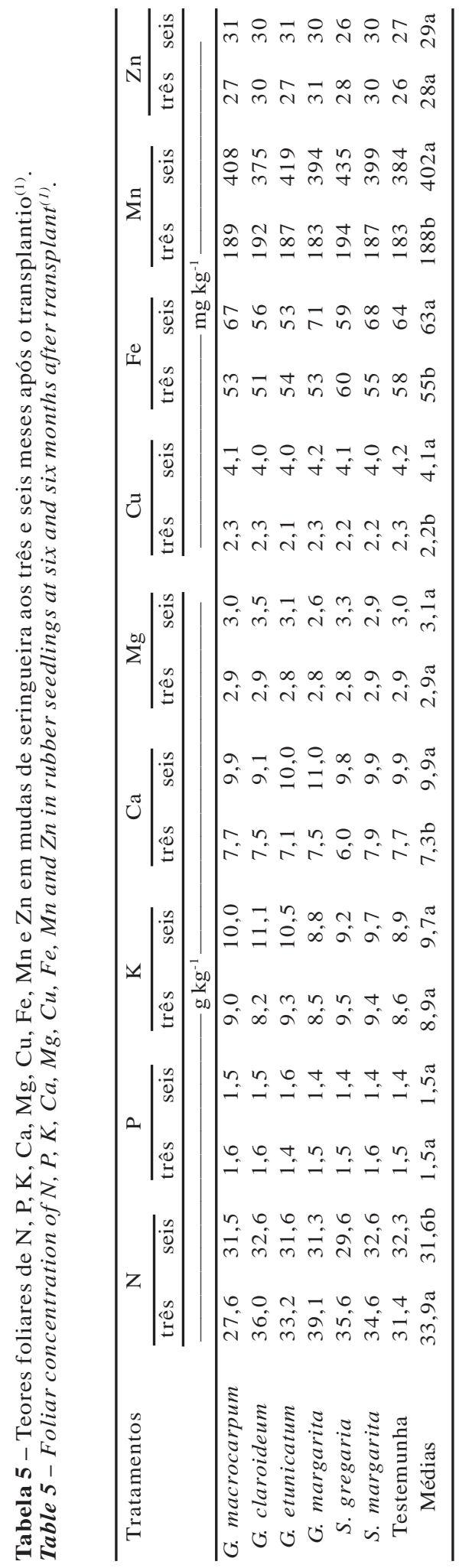

Á semelhante do P, os teores de K não apresentaram variação significativa ( $\mathrm{p}>0,05)$, em função das épocas de amostragem e da presença de FMAs (Tabela 4). Na média, os teores variaram de 8,2 a 11,1 $\mathrm{g} \mathrm{kg}^{-1}$, com a média de avaliação feita no sexto mês de $9,7 \mathrm{~g} \mathrm{~kg}^{-1}$, valor esse maior que os $8,9 \mathrm{~g} \mathrm{~kg}^{-1}$ obtidos no terceiro mês. Esse incremento no teor possivelmente se deva ao maior número de raízes adventícias, aumentando a absorção do nutriente no solo.

Resultados sobre o aumento expressivo dos teores foliares de Ca e Mg em plantas inoculadas com isolados fúngicos foram verificados por Silva et al. (2007b) em Eucalytus grandis. No caso da seringueira, tal fato não ocorreu. Os teores foliares de Ca e Mg nas plantas cultivadas nos solos com FMAs foram, em geral, semelhantes ao da testemunha (Tabela 5), havendo, no caso do Ca, diferenças (pd”0,05) entre as épocas de amostragem. Independentemente do tratamento, os teores de Ca variaram de 6,0 a 7,9 $\mathrm{g} \mathrm{kg}^{-1}$ no terceiro mês e de 9,1 a 11,0 $\mathrm{g} \mathrm{kg}^{-1}$ no sexto mês. Com relação ao $\mathrm{Mg}$, a variação foi de 2,8 a 2,9 $\mathrm{g} \mathrm{kg}^{-1}$ no terceiro mês e de 2,6 a 3,5 $\mathrm{g} \mathrm{kg}^{-1}$ no sexto mês. Na última avaliação, os teores ficaram acima de 7,6 a 8,2 g kg-1 e 1,7 a 2,4 $\mathrm{g} \mathrm{kg}^{-1}$, indicados como adequados para Ca e Mg, respectivamente (BOLLE-JONES, 1954).

Corroborando Lopes e Cardoso (2001), os teores foliares de $\mathrm{Cu}, \mathrm{Fe}$, Mn e Zn não foram influenciados pela presença no substrato de FMAs, havendo diferenças significativas do $\mathrm{Cu}$, Fe e Mn somente em função da época de amostragem (Tabela 5). No caso do Mn, outra hipótese da ausência de resultados foi que a grande absorção desse nutriente verificada com o alto teor foliar deve ter inibido a atuação dos FMAs na associação com as radicelas da seringueira (LINS et al, 2007). Na média, os teores de $\mathrm{Cu}, \mathrm{Fe}, \mathrm{Mn}$ e $\mathrm{Zn}$ variaram de 2,2 a 4,1 mg kg-1, 55 a $63 \mathrm{mg} \mathrm{kg}^{-1}, 188$ a $402 \mathrm{mg} \mathrm{kg}^{-1} \mathrm{e} 28$ a $29 \mathrm{mg} \mathrm{kg}^{-1}$, respectivamente. Exceto os de $\mathrm{Mn}$, os teores de $\mathrm{Cu}$, Fe e $\mathrm{Zn}$ ficaram abaixo dos indicados como adequados por Haag e Viégas (2000) e Moreira et al. (2006) para mudas de seringueira.

\section{CONCLUSÕES}

Assim, chegou-se ás seguintes conclusões:

- Em sistemas inalterados ou em monocultivo, a seringueira adulta não apresentou dependência obrigatória com FMAs.

Revista Árvore, Viçosa-MG, v.34, n.3, p.389-397, 2010 
- Seis meses de plantio foram suficientes para ocorrer colonização micorrízica detectável, com aumento no número de esporos nas mudas de seringueira, independentemente do inóculo utilizado.

- Os teores foliares de N, P, K, Ca, Mg, Cu, Fe, Mn e Zn não foram influenciados pelo número de esporos e grau de colonização de MVA, apenas pela idade das plantas.

\section{AGRADECIMENTOS}

Ao programa SHIFT [convênio Alemanha (Universidade de Bayreuth) - Brasil (Embrapa Amazônia Ocidental)] e ao CNPq, pelo suporte financeiro para realização do experimento e pela bolsa de estudos para o primeiro autor.

\section{REFERÊNCIAS}

ALVAREZ V., V. H. et al. Interpretação dos resultados das análises de solos. In: RIBEIRO, A. C. et al. (Eds.) Recomendação para o uso de corretivos e fertilizantes em Minas Gerais. Viçosa, MG: CFSEMG, 1987. p.25-32.

BOLLE-JONES, F. W. Nutrition of Hevea brasiliensis. Effect of nutrient deficiency on growth, chlorophyll, rubber and mineral contents of Tjirandjil seedlings. Journal of Rubber Research Institute of Malaysia, v.12, NUMERO, p.209-211, 1954.

DINIZ, P. F. A. et al. Crescimento, parâmetros biofísicos e anatômicos de plantas jovens de seringueira inoculadas com fungos micorrizicos arbusculares Glomus clarum. Acta Botanica Brasilica, v.24, n.1, p.65-72, 2010.

EMPRESA BRASILEIRA DE PESQUISA AGROPECUÁRIA - EMBRAPA. Centro Nacional de Pesquisa do Solo. Manual de métodos de análise de solo. Rio de Janeiro: 1997. 212p.

FELDMAN, F.; JUNQUEIRA, N. T. V.; LIBEREI, R. Utilization of VA-Mycorrhiza as a factor of integrated plant protection. In: EUROPEAN SYMPOSIUM ON MYCORRHIZA, 2.,1988, Praga. Anais.... Praga: European Society of Soil Science, 1988. 4p.

GASPAROTTO, L. et al. Doenças da seringueira no Brasil. Brasília: Embrapa-PI, 1997. 168p.

Revista Árvore, Viçosa-MG, v.34, n.3, p.389-397, 2010
GERDEMANN, J. W.; NICOLSON, T. H. Spores of mycorrhizal Endogone species extracted from soil by wet sieving and decanting. Transactions of the British Mycological Society, v.46, n.2, p.235-244, 1963.

GIOVANNETI, M.; MOSSE, B. Revolution of technique for measuring vesicular arbuscular mycorrhizae infection in roots. New

Phytopathology, v.84, n.3, p.489-500, 1980.

HAAG, H. P.; VIÉGAS, I. J. M. Crescimento e extração de nutrientes da seringueira. In: VIÉGAS, I. J. M.; CARVALHO, J. G. Seringueira; nutrição e adubação no Brasil. Brasília: Embrapa, 2000. p.77-121.

IKRAM, A.; MAHMUD, A. W. Endomycorrhizal fungi in soils under rubber. Journal Rubber Research Institute of Malaysia, v.32, n.2, p.198-206, 1984.

JENKINS, W. R. A rapid centrifugal-flotation technique for separating nematodes from soil. Plant Disease Reporter, v.48, n.9, p.692, 1964.

LINS, C. E. L. et al. Efeitos de fungos micorrízicos arbusculares no crescimento de mudas de Leucaena leucocephala (LAM) de WIT. em solos de caatinga sob impacto de mineração de cobre. Revista Árvore, v.31, n.2, p.355-363, 2007.

LOPES, O. M. N.; CARDOSO, E. J. B. N. Efeito do fósforo e da micorriza sobre o crescimento e a absorção de nutrientes por mudas de seringueira (Hevea barasiliensis Muel Arg.). Revista de Ciências Agrárias. v.35, v.1, p.41-53, 2001.

MARSCHNER, H.; DELL, B. Nutrient uptake in mycorrhizal symbiosis. Plant and Soil, v.59, n.1, p.89-102, 1994.

MORAES, V. H. F. Adubação de porta-enxertos de seringueira em sacos plásticos. In:

EMBRAPA. Síntese: tecnologias geradas pelo sistema. Brasília: EmbrapaDDT, 1984. p.206.

MORAES, V. H. F.; MORAES, L. A. C. Desempenho de clones de copa de seringueira resistentes ao mal-das-folhas. Pesquisa Agropecuária Brasileira, v.45, n.11, p.852-858, 2008. 
MOREIRA, A.; FAGERIA, N. K. Soil chemical attributes of Amazonas State, Brazil. Communications in Soil Science and Plant Analysis, v.40, n.17/18, p.2912-2925, 2009.

MOREIRA, A.; MORAES, L. A. C.; FAGERIA, N. K. Potential of rubber plantations for environmental conservation in amazon region. Bioremediation, Biodiversity and Bioavailability, v. 3, n.1, p.1-5, 2009.

MOREIRA, A.; MORAES, V. H. F.; CASTRO. C. Fontes e doses de boro em porta-enxertos de seringueira. Pesquisa Agropecuária Brasileira, v.41, n.8, p.1291-1298, 2006.

MORTON, M. Rubber technology. New York: Van Nostrand Reinhold, 1989. 74p.

NELSON, D. W.; SOMMERS, L. E. Determination of total nitrogen in plant material. Agronomy Journal, v.65, n.1, p.109-112, 1972.

NOVOZAMSKY, I. et al. novel digestion technique for multi-element plant analysis. Communications in Soil Science and Plant Analysis, v.14, n.3, p.239-248, 1983.

OLIVEIRA, A. N.; OLIVEIRA, L. A. Colonização por fungos micorrízicos arbusculares e teores de nutrientes em cinco cultivares de bananeiras em um Latossolo da Amazônia. Revista Brasileira de Ciência do Solo, v.29, n.3, p.481-488, 2005.

OLIVEIRA, L. A.; GUITTON, T. L.; MOREIRA, F. W. Relações entre as colonizações por fungos micorrízicos arbusculares e teores de nutrientes foliares em oito espécies florestais da Amazônia. Acta Amazonica, v.29, n.2, p.183-193, 1999.

PEREIRA, E. B. C.; PEREIRA, A. V. P.; SILVA, S. E. L. Doses de $\mathbf{N}, \mathbf{P}, \mathrm{K}$ e Mg para viveiro de seringueira em Latossolo Amarelo de textura muito argilosa, em Manaus. Manaus: CNPSD, 1986. 10p.

PHILLIPS, J. M.; HAYMAN, D. S. Improved procedures for clearing roots and staining parasitic and vesiculararbuscular mycorrhizal fungi for assessment of infection. Transactions of the British Mycological Society, v.55, n.1, p.158-161, 1970.
PIMENTEL GOMES, F.; GARCIA, C. H. Estatística aplicada a experimentos agronômicos e florestais. Piracicaba: FEALQ, 2002. 309p.

SANCHEZ, P. A. et al. Amazon basin soils: management for continuous crop production. Science, v.216, n.4548, p.821-827, 1982.

SILVA, L. X. et al. Fungos micorrízicos arbusculares em áreas de plantio de leucena e sabiá no Estado de Pernambuco. Revista Árvore, v.31, n.3, p.427-435, 2007a.

SILVA, M. A. et al. Formação de ectomicorrizas por monocários e dicários de Pisolithus sp. e interações nutricionais em Eucalyptus grandis. Revista Brasileira de Ciência do Solo, v.31, n.5, p.917-929, 2007b.

SIQUEIRA, J. O. Avanços em fundamentos e aplicação de micorrizas. Lavras: DCSDCF/UFLA, 1996. 290p.

TAWARAYA, K. et al. Arbuscular mycorrhizal colonization of three species grown in peat swamp of Central Kalimantan Indonesia. Forest Ecology and Management, v.182, n.2, p.381-386, 2003.

VIEIRA, L. S.; SANTOS, P. C. T. C. Amazônia; seus solos e outros recursos naturais. São Paulo: Agronômica Ceres, 1987. 416p.

WALINGA, I. et al. Plant analysis manual. Dordrecht: Kluwer Academic Publishers. 1995. 129p.

WASTIE, R. L. The occurrence of an endogene type of endotrophic mycorrhiza in Hevea brasiliensis. Transactions of the British Mycological Society, v.48, n.1, p.167-178, 1965.

WALLANDER, H.; JOHANSSON, L.; PALLON, J. PIXEL analysis to estimate the composition of ectomycorrhizal rhizomorphs grow in contact with different minerals in forest soil. FEMS Microbiology Ecology, v.39, n.1, p.147-146, 2002.

Revista Árvore, Viçosa-MG, v.34, n.3, p.389-397, 2010 